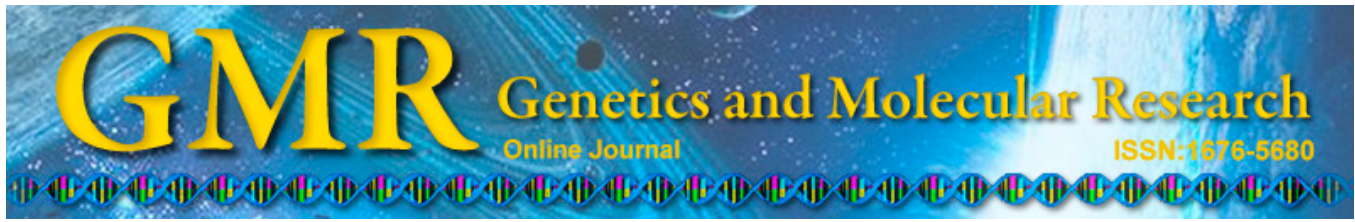

\title{
Hypoxia induces dysregulation of local renin-angiotensin system in mouse Lewis lung carcinoma cells
}

\author{
L. Fan*, Y. Feng*, H.Y. Wan, L. Ni, Y.R. Qian, Y. Guo, Y. Xiang and Q.Y. Li \\ Department of Respiration, Ruijin Hospital, \\ Shanghai Jiaotong University School of Medicine, Shanghai, China \\ *These authors contributed equally to this study. \\ Corresponding author: H.Y. Wan \\ E-mail: wanhuanyingruijin@gmail.com
}

Genet. Mol. Res. 13 (4): 10562-10573 (2014)

Received January 15, 2014

Accepted May 1, 2014

Published December 12, 2014

DOI http://dx.doi.org/10.4238/2014.December.12.19

\begin{abstract}
The renin-angiotensin system (RAS) influences cancer biology and is frequently dysregulated in malignancy. However, regulation of tumor local RAS remains poorly understood. Hypoxia is a hallmark of solid tumors and affects nearly every major aspect of cancer biology. Previous studies have shown that hypoxia can regulate RAS expression in somatic tissues and cells. The aim of this study was to investigate the influence of hypoxia on local RAS expression in mouse Lewis lung carcinoma (LLC) cells. For hypoxia treatment, LLC cells were cultured in a hypoxia incubator or treated with hypoxia-mimetic cobalt chloride. Hypoxia up-regulated angiotensin II, angiotensinconverting enzyme (ACE), and angiotensin II type 1 receptor (AT1R), and down-regulated ACE2 and angiotensin II type 2 receptor in LLC cells. Captopril, an ACE inhibitor, and losartan, an AT1R blocker, decreased expression of ACE and AT1R, but increased expression of ACE2 and angiotensin II type 2 receptor in LLC cells under hypoxia. Captopril and losartan also suppressed vascular endothelial growth factor-A expression in LLC cells under hypoxia. These findings suggest
\end{abstract}


that hypoxia induces dysregulation of local RAS in LLC cells. The pathophysiological importance of hypoxia-induced RAS dysregulation and potentially therapeutic effects of RAS inhibitors on hypoxic tumor cells should be further examined.

Key words: Renin-angiotensin system; Lewis lung carcinoma cell; Hypoxia; Vascular endothelial growth factor-A

\section{INTRODUCTION}

Lung cancer is one of the leading causes of cancer deaths worldwide (Jemal et al., 2011). Despite numerous advances in diagnosis and treatment, the prognosis for lung cancer remains poor. An increased understanding of lung cancer biology and new therapeutic targets are urgently needed.

The renin-angiotensin system (RAS) regulates cardiovascular homoeostasis, cell proliferation, and inflammation (Fyhrquist and Saijonmaa, 2008). Accumulating molecular and in vivo evidence indicates that the expression and activity of RAS contribute to the progression of malignancy (George et al., 2010; Gallagher et al., 2011). Drugs that inhibit RAS may retard tumor growth and metastasis (Suganuma et al., 2005; Attoub et al., 2008; Neo et al., 2010).

Most components of the RAS, including angiotensinogen, angiotensin-converting enzyme (ACE), and angiotensin receptors are expressed locally in a wide variety of tumors (George et al., 2010). ACE converts the inactive pro-hormone angiotensin I (ANG I) to the active peptide hormone angiotensin II (ANG II), which is the main effecter peptide of the RAS. The effects of ANG II are mainly mediated by the ANG II receptors angiotensin II type 1 receptor (AT1R) and angiotensin II type 2 receptor (AT2R). ANG II promotes tumor proliferation, migration, angiogenesis, and metastasis primarily through AT1R (Egami et al., 2003; Fujita et al., 2002, 2005). AT2R may counter the activities of AT1R (Pickel et al., 2010). However, AT2R can also promote tumor angiogenesis (Pickel et al., 2010) and proliferation (Kanehira et al., 2005). In addition, the RAS includes ACE2 (Fleming et al., 2005), which converts ANG I or ANG II to angiotensin 1-7 (ANG 1-7). ANG 1-7 exerts opposite actions to the ANG II through its specific mas receptor (Iwai and Horiuchi, 2009). In contrast to ACEANG II-AT1R axis, the ACE2-ANG 1-7-mas axis inhibits tumor proliferation, angiogenesis, tumor cell migration, and invasion (Gallagher and Tallant, 2004; Soto-Pantoja et al., 2009; Ni et al., 2012).

Dysregulation of RAS components is frequently observed in malignancy, including in lung cancer (Feng et al., 2010). Imbalanced RAS components contribute to tumor growth, angiogenesis, and metastasis and are correlated with prognosis (Suganuma et al., 2005; Rocken et al., 2007; Kinoshita et al., 2009; Feng et al., 2010; Neo et al., 2010). However, the regulation of tumor local RAS remains poorly understood.

Hypoxia is a hallmark of solid tumors and affects nearly every major aspect of cancer biology. Hypoxic areas are frequently observed in solid tumors because of uncontrolled tumor growth and aberrant tumor vasculature. Previous studies have shown that hypoxia can regulate RAS expression in somatic tissues and cells (Fung et al., 2002; Zhang et al., 2009; Mao et al., 2010). It has also been shown that target RAS attenuates hypoxia-induced cell proliferation and migration (Zhang et al., 2009). These observations suggest that hypoxia is a driving force 
in the dysregulation of tumor local RAS. However, the relevance of hypoxia to RAS expression in malignancy has not been thoroughly examined.

In this study, we found that hypoxia up-regulated ANG II, ACE, and AT1R, while it down-regulated ACE2 and AT2R in Lewis lung carcinoma (LLC) cells. Captopril, an ACE inhibitor, and losartan, an AT1R blocker, decreased the expression of ACE and AT1R and increased the expression of ACE2 and AT2R in LLC cells under hypoxia conditions. Captopril and losartan also suppressed vascular endothelial growth factor (VEGF)-A expression in LLC cells under hypoxia. These findings suggest that hypoxia induces dysregulation of local RAS in LLC cells. The pathophysiological importance of hypoxia-induced RAS dysregulation and potentially therapeutic effects of RAS inhibitors on hypoxic tumor cells should be further examined.

\section{MATERIAL AND METHODS}

\section{Cell culture and hypoxia exposure}

Mouse LLC cells were obtained from the Cell Bank of Chinese Academic of Sciences, Shanghai, China. LLC cells were cultured in Dulbecco's modified Eagle's medium (Gibco, Grand Island, NY, USA) supplemented with 10\% fetal bovine serum (Gibco), and incubated in humidified air at $5 \% \mathrm{CO}_{2}$ and $37^{\circ} \mathrm{C}$.

We applied 2 strategies for hypoxia treatment: 1) LLC cells were cultured in a hypoxia incubator (Heraeus Instruments, Hanau, Germany) under hypoxic conditions $\left(1 \% \mathrm{O}_{2}, 94 \% \mathrm{~N}_{2}\right.$, $\left.5 \% \mathrm{CO}_{2}\right)$ for $0,6,12$, and $24 \mathrm{~h}$. 2) LLC cells were treated with different concentrations $(0,50$, and $100 \mu \mathrm{M}$ ) of $\mathrm{CoCl}_{2}$, a hypoxic-mimetic agent, under normoxic conditions for $24 \mathrm{~h}$.

\section{Detection of ANG II concentration}

LLC cells were plated at $2 \times 10^{5}$ cells per well on 6-well plates. When confluent, the cells were incubated with fresh medium and cultured under normoxic conditions or hypoxia conditions $\left(1 \% \mathrm{O}_{2}\right.$ in a hypoxia incubator or treated with $\left.100 \mu \mathrm{M} \mathrm{CoCl}_{2}\right)$. After $24 \mathrm{~h}$, the cell culture supernatants were aspirated and the cells were scraped from the culture dishes with protein lysis buffer. The ANG II concentration in the culture supernatants and cellular extract was detected using an ANG II radioimmunoassay at Ruijin Hypertension Institute Laboratory, Shanghai, China. Intracellular ANG II concentration was related to total cellular protein, which was determined using a BCA kit (Beyotime, Shanghai, China).

\section{Real-time quantitative reverse transcription (qRT) analysis}

ACE, ACE2, AT1R (AT1aR, AT1bR), and AT2R mRNA expression was examined by real-time qRT-based polymerase chain reaction (qRT-PCR) in LLC cells. RNA was extracted from cells using TRIzol reagent (Invitrogen, Carlsbad, CA, USA) and treated with DNase (Promega, Madison, WI, USA). Complementary DNA was synthesized using a cDNA synthesis kit (Applied Biosystems, Foster City, CA, USA). Fluorescence qRT-PCR was performed using a SYBR green I kit (TaKaRa Biotechnology, Shiga, Japan), using the ABI PRISM 7900 system (Applied Biosystems). The SYBR Green assay PCR was initiated with 1 cycle at $95^{\circ} \mathrm{C}$ for $30 \mathrm{~s}$, followed by 40 cycles at $95^{\circ} \mathrm{C}$ for $5 \mathrm{~s}$, and at $60^{\circ} \mathrm{C}$ for $30 \mathrm{~s}$. 
All data were analyzed using the ABI PRISM SDS 2.0 software (PerkinElmer, Waltham, MA, USA). Using the $\Delta \mathrm{Ct}$ method, $\beta$-actin was co-amplified to normalize the amount of RNA added to the reaction, and the data were subjected to cycling threshold analysis. PCR was performed in triplicate, and standard deviations representing experimental errors were calculated. The following primers used for LLC cells: $\beta$-actin: forward (5'-TGACAGGATGCAGAAGG AGA-3'), reverse (5'-GCTGGAAGGTGGACAGTGAG-3'); ACE: forward (5'-AAGCCTAC AGACAAGACG-3'), reverse (5'-CCAGCGAGGTGAAGAA-3'); ACE2: forward (5'-CCCAT TTGCTTGGTGATA-3'), reverse (5'-CATTGGCTCCGTTTCTTA-3'); AT1aR: forward (5'-TTGTCCACCCGATGAAGT-3'), reverse (5'-TGGCAGTGTCCACGATGT-3'); AT1bR: forward (5'-TTTTGGACCTGTGATGT-3'), reverse (5'-GTTGGCAATGAGGATAG-3'); AT2R: forward (5'-TTTTGGACCTGTGATGT-3'), reverse (5'-GTTGGCAATGAGGATAG-3').

\section{Western blot analysis}

Hypoxia-inducible factor (HIF)-1 $\alpha$, ACE, ACE2, AT1R, and AT2R protein levels in LLC cells were detected. After treatment with a protein assay reagent (Bio-Rad Laboratories, Hercules, CA, USA), soluble protein was separated on $8 \%$ sodium dodecyl sulfate-polyacrylamide gels and transferred to polyvinylidene fluoride membranes (Millipore, Billerica, MA, USA). The membranes were blocked with a 5\% nonfat dry milk solution in Tris-buffered

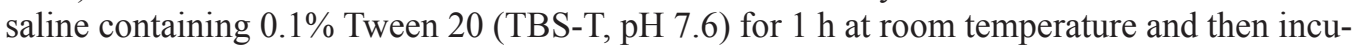
bated in primary antibody in blocking solution at $4^{\circ} \mathrm{C}$ overnight. The HIF-1 $\alpha$, AT1R, AT2R (1:500; Abbiotec, San Diego, CA, USA), ACE, ACE2 (1:500; R\&D Systems, Minneapolis, MN, USA), and $\beta$-actin (1:5000; Sigma, St. Louis, MO, USA) antibodies were used. After the membranes were washed 3 times for $5 \mathrm{~min}$ in Tris-buffered saline containing $0.1 \%$ Tween 20 , they were incubated with horseradish peroxidase-conjugated secondary antibody (1:2000, Santa Cruz Biotechnology, Santa Cruz, CA, USA) in blocking buffer for $2 \mathrm{~h}$ at room temperature. Immunoreactive bands were visualized using ECL Plus Reagent (Amersham Biosciences, Amersham, UK).

\section{Statistical analysis}

Values are reported as means \pm standard deviation (SD). Statistical comparisons were made using the Student $t$-test. $\mathrm{P}<0.05$ was considered to be statistically significant.

\section{RESULTS}

\section{Effects of hypoxia on ANG II expression in LLC cells}

LLC cells were cultured under normoxia or hypoxia conditions $\left(1 \% \mathrm{O}_{2}\right.$ in a hypoxia incubator or treated with $100 \mu \mathrm{M} \mathrm{CoCl}_{2}$ ) for $24 \mathrm{~h}$. Optical microscopy showed that LLC cells exposed to hypoxia had a larger cell volume than those cultured under normoxia conditions. In addition, HIF-1 $\alpha$ protein accumulated in LLC cells exposed to hypoxia. ANG II concentrations measured by ANG II radioimmunoassay in supernatants and cellular extract were higher under hypoxia than under normoxia conditions (Figure 1). 


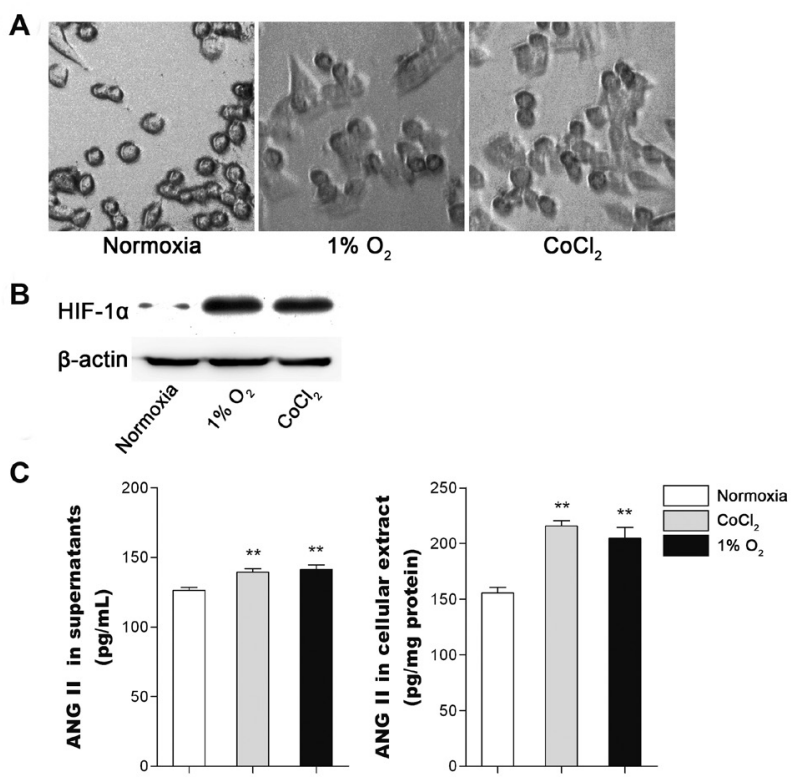

Figure 1. Hypoxia enhances ANG II expression in LLC cells. LLC cells were cultured under normoxia or hypoxia (under $1 \% \mathrm{O}_{2}$ or treated with $100 \mu \mathrm{M} \mathrm{CoCl}_{2}$ ) for $24 \mathrm{~h}$. A. Effects of hypoxia on morphology of LLC cells observed under optical microscope (100X). B. Western blot of hypoxia-inducible factor- $1 \alpha$ (HIF-1 $\alpha$ ), with $\beta$-actin as a loading control. C. ANG II concentration measured by RIA in supernatants and in cellular extract normalized against total cellular protein of LLC cells. Values are reported as means $\pm \mathrm{SE}(\mathrm{N}=6)$. $* * \mathrm{P}<0.01$ vs normoxia.

\section{Hypoxia up-regulated ACE and down-regulated ACE2 in LLC cells}

LLC cells were incubated under $1 \% \mathrm{O}_{2}$ for $0,6,12$, and $24 \mathrm{~h}$. Real-time PCR analysis showed that ACE mRNA was markedly increased, while ACE2 mRNA markedly decreased in LLC cells during hypoxia, both showing time-dependent regulation by hypoxia. The ACE protein was increased, while the ACE2 protein was decreased during hypoxia in LLC cells (Figure 2). LLC cells were also treated with different concentrations $(0,50$, and $100 \mu \mathrm{M})$ $\mathrm{CoCl}_{2}$ for $24 \mathrm{~h} . \mathrm{CoCl}_{2}$ dose-dependently increased ACE mRNA and protein levels while it decreased ACE2 mRNA and protein levels (Figure 3). The ratios of ACE/ACE2 protein levels were significantly increased in LLC cells exposed to hypoxia.

\section{Hypoxia up-regulated AT1R and down-regulated AT2R in LLC cells}

Real-time PCR analysis revealed a marked increase in AT1aR and AT2R mRNA expression, but no significant changes in AT1bR mRNA expression under hypoxic exposure (Figures 2 and 3). Repeated examination showed that AT1R and AT2R proteins were regulated in a diphase manner in LLC cells exposed to $1 \% \mathrm{O}_{2}$ at various time points. During the early phase $(6 \mathrm{~h})$, the AT1R protein was decreased, while the AT2R protein was increased. As hypoxia continued, the AT1R protein was significantly increased and the AT2R protein was significantly decreased (Figure 2). Treatment with $\mathrm{CoCl}_{2}$ for $24 \mathrm{~h}$ significantly increased the AT1R protein and decreased the AT2R protein (Figure 3). 
A
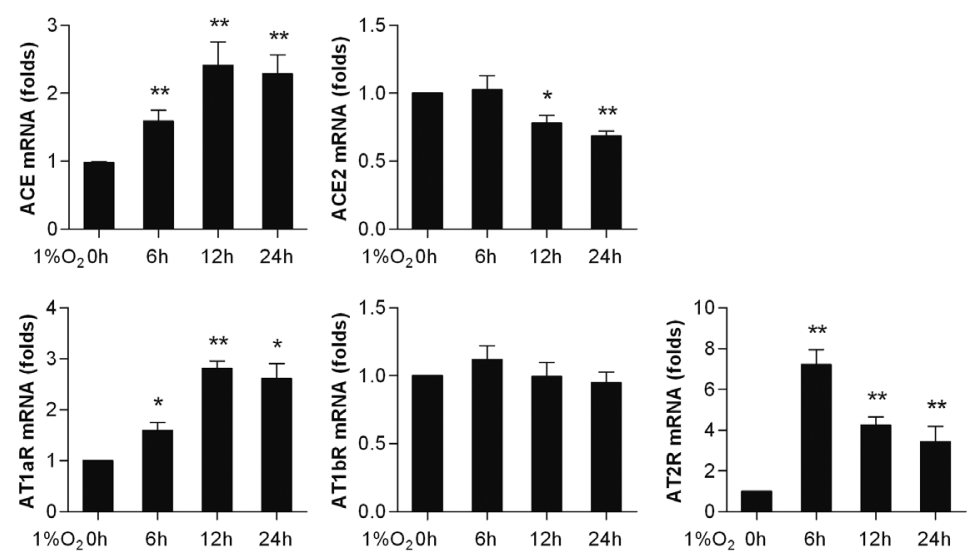

B

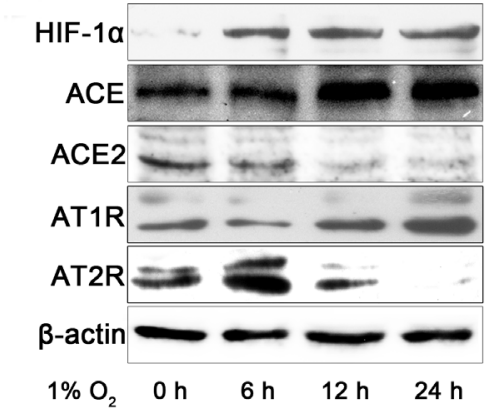

C
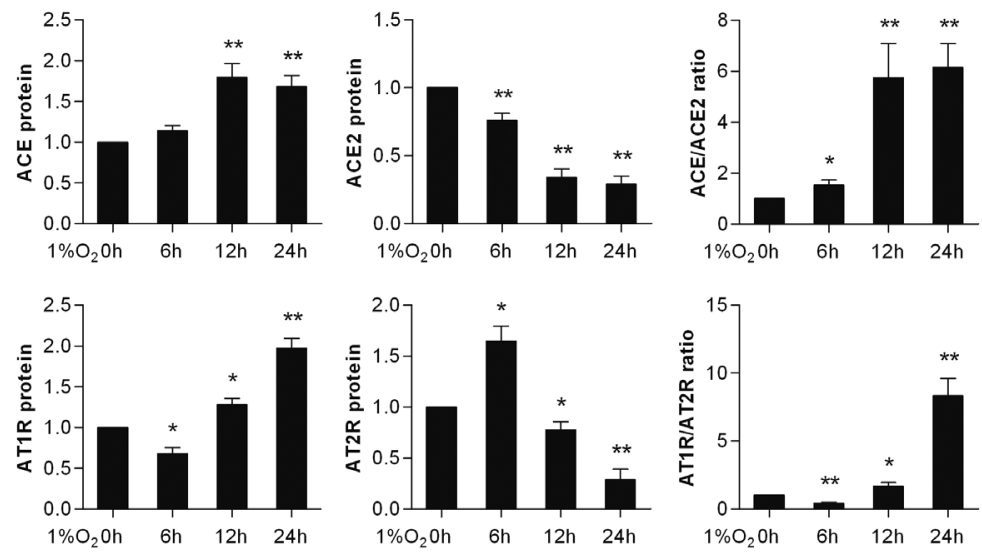

Figure 2. Effects of hypoxia $\left(1 \% \mathrm{O}_{2}\right)$ on RAS expression in LLC cells. LLC cells were exposed to $1 \% \mathrm{O}_{2}$ for 0,6 , 12, and $24 \mathrm{~h}$. A. Real-time quantitative RT-PCR of mRNA levels of ACE, ACE2, AT1R (AT1aR and AT1bR), and AT2R in LLC cells with $\beta$-actin as an internal control. B. Western blot of HIF-1 $\alpha$, ACE, ACE2, AT1R, and AT2R proteins in LLC cells with $\beta$-actin as a loading control. C. Mean data for ACE, ACE2, AT1R, and AT2R protein expression. Values in $(\mathrm{A})$ and $(\mathrm{C})$ are fold-increases with $0 \mathrm{~h}$ value as 1 . All experiments were repeated in triplicate with similar results. $* \mathrm{P}<0.05$ and $* * \mathrm{P}<0.01$ vs $0 \mathrm{~h}$. 
A
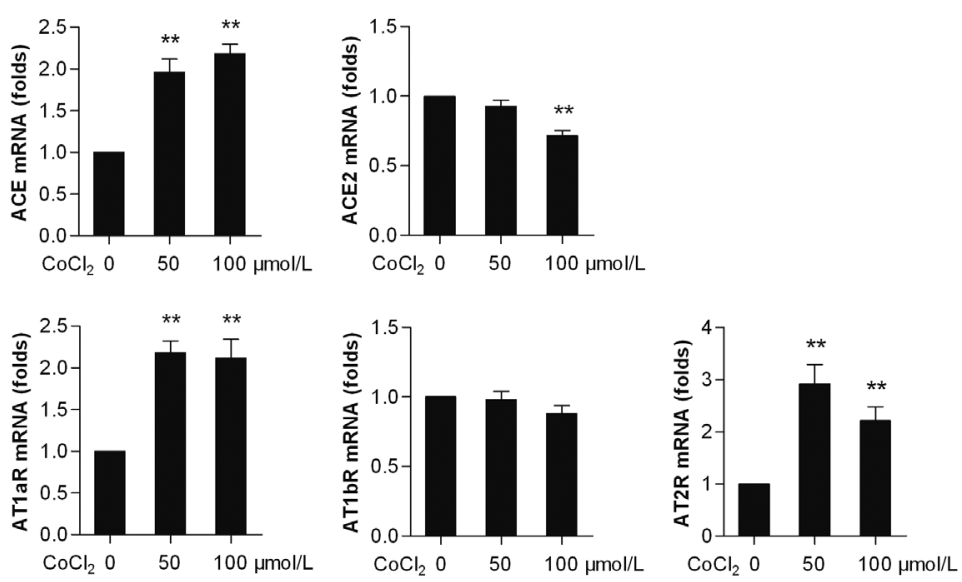

B

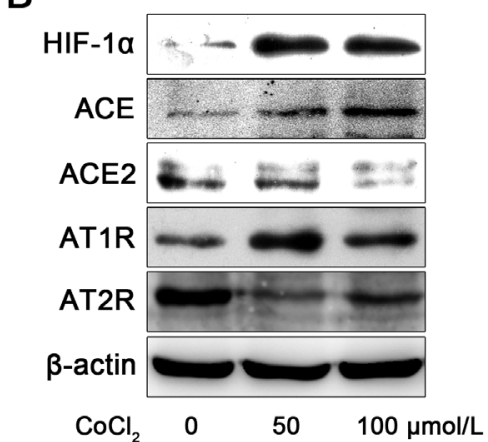

C
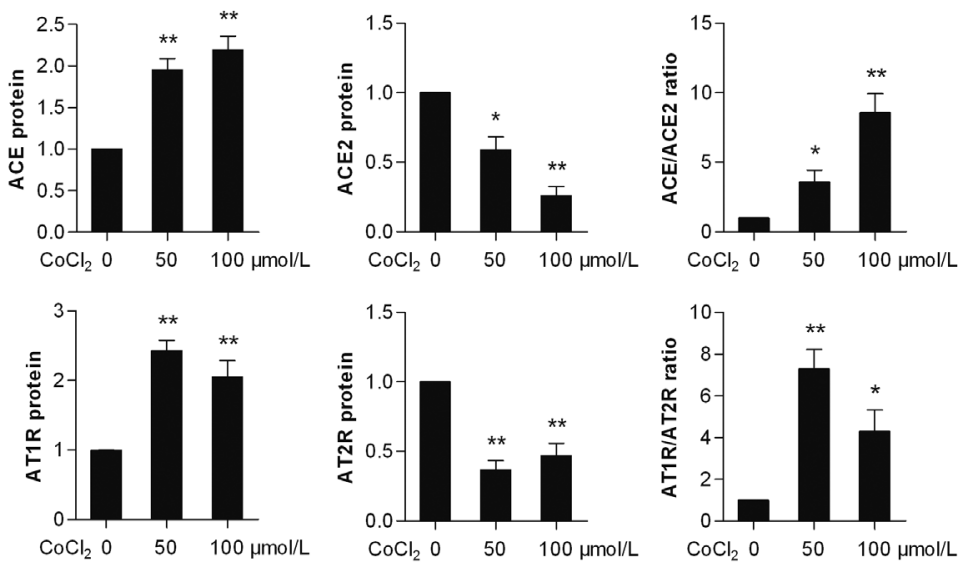

Figure 3. Effects of hypoxia $\left(\mathrm{CoCl}_{2}\right)$ on RAS expression in LLC cells. LLC cells were treated with 0,50 and $100 \mu \mathrm{M} \mathrm{CoCl}_{2}$ for $24 \mathrm{~h}$. A. Real-time quantitative RT-PCR of mRNA levels of ACE, ACE2, AT1R (AT1aR and AT1bR), and AT2R in LLC cells with $\beta$-actin as an internal control. B. Western blot of HIF-1 $\alpha$, ACE, ACE2, AT1R, and AT2R proteins in LLC cells with $\beta$-actin as a loading control. C. Mean data for ACE, ACE2, AT1R, and AT2R protein expression. Values in (A) and (C) are fold-increases, with $0 \mu \mathrm{M}$ value as 1 . All experiments were repeated in triplicate with similar results. $* \mathrm{P}<0.05$ and $* * \mathrm{P}<0.01$ vs $0 \mu \mathrm{M}$. 


\section{RAS inhibitors modulated RAS expression in LLC cells exposed to hypoxia}

We tested the effects of captopril, an ACE inhibitor, and losartan, an AT1R blocker, on the expression of RAS components in LLC cells exposed to hypoxia. LLC cells incubated under $1 \% \mathrm{O}_{2}$ were treated with captopril $(100 \mu \mathrm{M})$ or losartan $(10 \mu \mathrm{M})$ for $24 \mathrm{~h}$. Captopril and losartan down-regulated ACE and AT1R proteins while they up-regulated ACE2 and AT2R proteins in LLC cells under hypoxia (Figure 4).

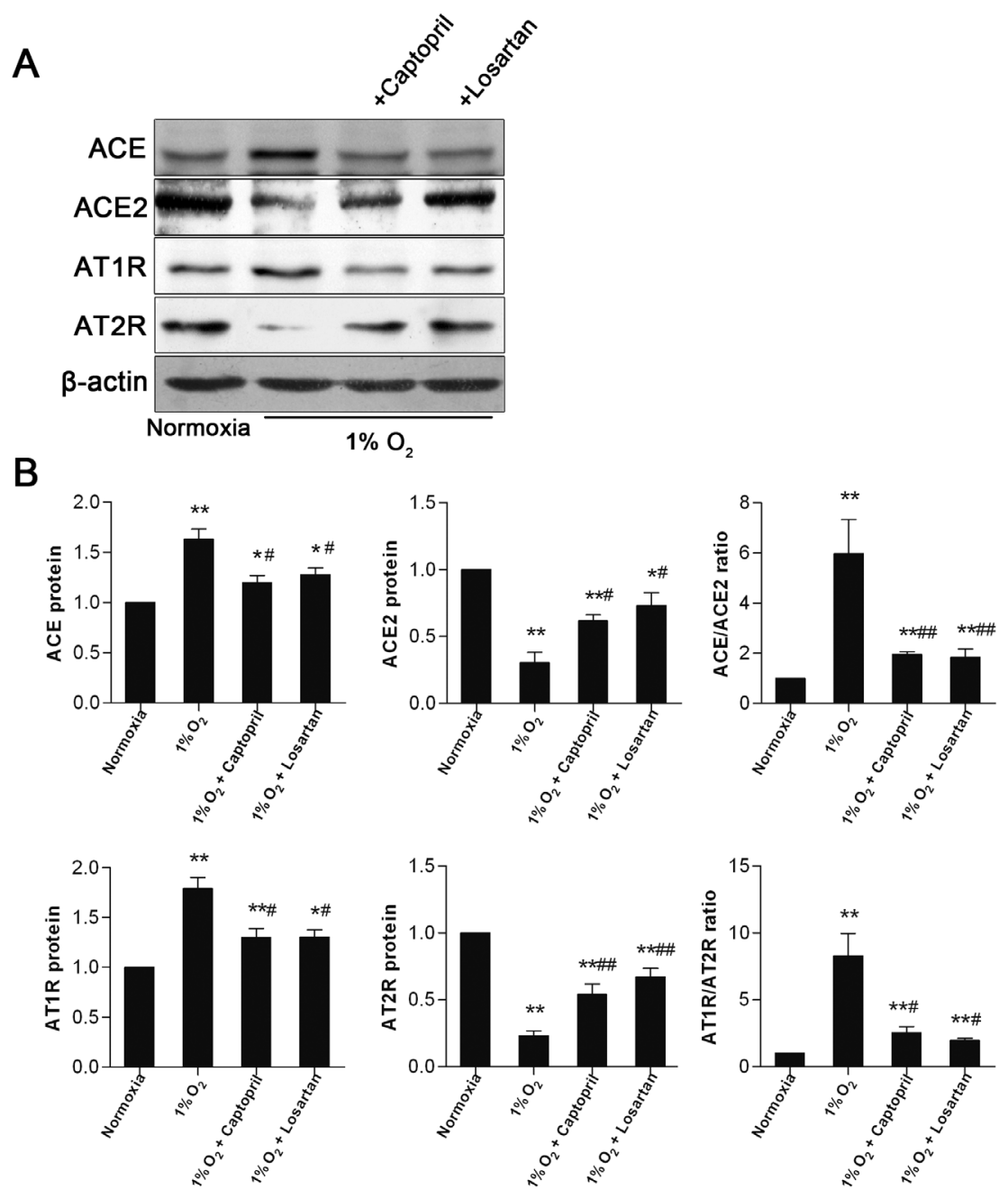

Figure 4. Effects of RAS inhibitors on RAS expression in LLC cells exposed to hypoxia. LLC cells were cultured under normoxia or hypoxia $\left(1 \% \mathrm{O}_{2}\right)$ in the presence of 0 or $100 \mu \mathrm{M}$ captopril or $10 \mu \mathrm{M}$ losartan for $24 \mathrm{~h}$. A. Western blot of ACE, ACE2, AT1R, and AT2R proteins. B. Mean data for ACE, ACE2, AT1R, and AT2R protein expression. Values (means $\pm \mathrm{SE}$ ) are fold-increases, with normoxia value as 1 . All experiments were repeated in triplicate with similar results. ${ }^{*} \mathrm{P}<0.05$ and ${ }^{* *} \mathrm{P}<0.01$ vs normoxia. ${ }^{\#} \mathrm{P}<0.05$ and ${ }^{\# \#} \mathrm{P}<0.01$ vs hypoxia without RAS inhibitors. 


\section{RAS inhibitors down-regulated VEGF-A in LLC cells exposed to hypoxia}

LLC cells under $1 \% \mathrm{O}_{2}$ were treated with $100 \mu \mathrm{M}$ captopril or $10 \mu \mathrm{M}$ losartan for $24 \mathrm{~h}$. Captopril and losartan down-regulated the VEGF-A protein in LLC cells under hypoxia (Figure 5).

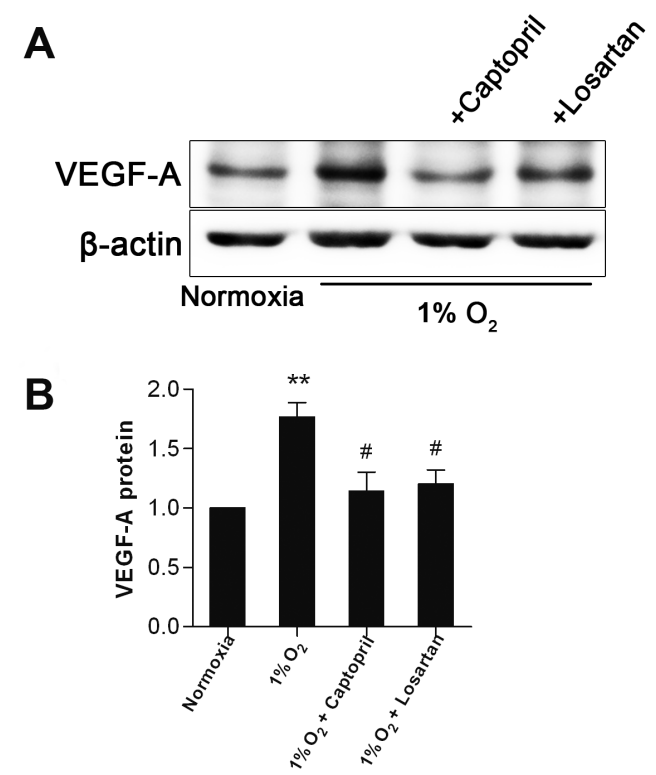

Figure 5. Effects of RAS inhibitors on VEGF-A expression in LLC cells exposed to hypoxia. LLC cells were cultured under normoxia or hypoxia $\left(1 \% \mathrm{O}^{2}\right)$ in the presence of 0 or $100 \mu \mathrm{M}$ captopril or $10 \mu \mathrm{M}$ losartan for 24 h. A. Western blot of the VEGF-A protein. B. Mean data for VEGF-A protein expression. Values (means $\pm \mathrm{SE}$ ) are fold increases, with normoxia value as 1 . All experiments were repeated in triplicate with similar results. $* * \mathrm{P}<0.01$ vs normoxia. ${ }^{\#} \mathrm{P}<0.05$ and ${ }^{\#} \mathrm{P}<0.01$ vs hypoxia without RAS inhibitors.

\section{DISCUSSION}

Tumor local RAS is becoming increasingly recognized as an important element of the tumor micro-environment, but regulation of tumor local RAS is not well-understood. The present study suggests that hypoxia is a driving force of dysregulation of local RAS in LLC cells.

ANG II is the main effecter peptide of RAS. In addition to circulatory RAS, ANG II is synthesized in local tissues (Van Kats et al., 1998). We found that hypoxia enhances local synthesis of ANG II in LLC cells. The balance between ACE and ACE2 is crucial for controlling ANG II levels. ACE converts ANG I to ANG II, while ACE2 converts ANG I or ANG II to ANG 1-7. Hypoxia exposure markedly increases ACE mRNA and protein levels, but decreases ACE2 mRNA and protein levels in LLC cells. We hypothesized that the imbalance of ACE/ACE2 participates in the up-regulation of ANG II induced by hypoxia. ANG II binds 2 major receptors: AT1R and AT2R. We observed that AT1R and AT2R proteins were regulated in a diphase manner during hypoxia. During the early phase, the AT1R protein was decreased while the AT2R protein was increased. As hypoxia continued, the AT1R protein increased and the AT2R protein decreased.

Complex cross-talk exists in RAS regulation. The ANG II-AT1R pathway up-regulates 
ACE, but down-regulates ACE2 (Koka et al., 2008). ANG II up-regulates AT1R and AT2R (Shibata et al., 1997). AT1R and AT2R inhibits the expression of each other (Tanaka et al., 1999; Volpe et al., 2003). We found that captopril and losartan up-regulated ACE2 and AT2R proteins in LLC cells under hypoxia, indicating that the ACE-ANG II-AT1R axis is involved in the down-regulation of ACE2 and AT2R. Captopril and losartan also down-regulated ACE and AT1R proteins in LLC cells under hypoxia, which may have resulted from elevated ACE2 and AT2 down-regulated ANG II and AT1R. A probable bi-directional control mechanism of AT1R and AT2R is that hypoxia enhances the expression of AT1R and AT2R via ANG II, but elevated AT2R inhibits AT1R expression during the early stage. As hypoxia continues, elevated AT1R then inhibits AT2R expression.

In the present study, we found that hypoxia enhanced the expression of the ACE-ANG II-AT1R axis, but inhibited the expression of ACE2 and AT2R in LLC cells. The ACE-ANG II-AT1R axis and ACE2-ANG (1-7)-mas axis play antagonistic roles (Iwai and Horiuchi, 2009). ANG II mainly acts through AT1R to regulate tumor cell proliferation, migration, and angiogenesis (Egami et al., 2003; Fujita et al., 2002, 2005; Suganuma et al., 2005). In contrast to the ACE-ANG II-AT1R axis, the ACE2-ANG (1-7)-mas axis inhibits tumor cell proliferation, migration, and invasion (Gallagher and Tallant, 2004; Soto-Pantoja et al., 2009; Ni et al., 2012). The second major ANG II receptor, AT2R, has been shown to antagonize the function of AT1R. AT2R over-expression effectively attenuates the growth of lung adenocarcinoma cells through intrinsic apoptosis (Pickel et al., 2010). However, AT2R may also promote tumorigenesis and cell proliferation (Kanehira et al., 2005; Pickel et al., 2010). Thus, the potential role of hypoxia-induced RAS dysregulation should be examined.

Hypoxia plays a crucial role in the development of solid tumors. HIF-1 $\alpha$ is the most important transcription factor for this process, and has been suggested as a target for cancer therapy. However, local RAS, as a signaling pathway downstream from hypoxia/HIF-1 $\alpha$, may also be an attractive therapeutic target. VEGF-A is a key regulator of tumor growth and tumorassociated angiogenesis, and is regulated via various signaling transduction pathways, including the ANG II-AT1R pathway (Imai et al., 2007). Captopril and losartan down-regulated VEGF-A in LLC cells under hypoxia. This suggests that RAS may play an important role in the hypoxia-induced pathophysiological response in lung cancer cells and may be a potential target to hypoxic tumor cells.

RAS inhibitors, such as ACE inhibitors and AT1R blockers, are widely used antihypertensive agents. It is unlikely that RAS inhibitors will be a single drug treatment for lung cancer. However, these agents may provide synergistic effects to conventional anti-tumor treatment and may be effective against hypoxic cancer cells. Additionally, RAS inhibitors not only directly inhibit ANG II-AT1R signaling, but also modulate expression of tumor local RAS and HIF-1 $\alpha$.

There were some limitations to our study. In the present study, we only focused on the expression of tumor local RAS components; however, we did not examine the mechanism of RAS dysregulation and the related downstream targets of RAS. Further, our results should be confirmed through animal experiments.

In summary, hypoxia enhanced the expression of the ACE-ANG II-AT1R axis, but inhibited the expression of ACE2 and AT2R in LLC cells. RAS inhibitors, both ACE inhibitors and AT1R blockers, modulate the expression of RAS components. These findings contribute to the understanding of tumor local RAS. The pathophysiological importance of hypoxiainduced RAS dysregulation and potentially therapeutic effects of RAS inhibitors on hypoxic tumor cells require further examination. 


\section{ACKNOWLEDGMENTS}

Research supported by the National Natural Science Foundation of China (\#81071925) and the Doctoral Fund of Medical School of Shanghai Jiaotong University (\#BXJ201109).

\section{REFERENCES}

Attoub S, Gaben AM, Al-Salam S, Al Sultan MA, et al. (2008). Captopril as a potential inhibitor of lung tumor growth and metastasis. Ann. N. Y. Acad. Sci. 65-72.

Egami K, Murohara T, Shimada T, Sasaki K, et al. (2003). Role of host angiotensin II type 1 receptor in tumor angiogenesis and growth. J. Clin. Invest. 112: 67-75.

Feng Y, Wan H, Liu J, Zhang R, et al. (2010). The angiotensin-converting enzyme 2 in tumor growth and tumor-associated angiogenesis in non-small cell lung cancer. Oncol. Rep. 23: 941-948.

Fleming I, Kohlstedt K and Busse R (2005). New fACEs to the renin-angiotensin system. Physiology 20: 91-95.

Fujita M, Hayashi I, Yamashina S, Itoman M, et al. (2002). Blockade of angiotensin AT1a receptor signaling reduces tumor growth, angiogenesis, and metastasis. Biochem. Biophys. Res. Commun. 294: 441-447.

Fujita M, Hayashi I, Yamashina S, Fukamizu A, et al. (2005). Angiotensin type 1a receptor signaling-dependent induction of vascular endothelial growth factor in stroma is relevant to tumor-associated angiogenesis and tumor growth. Carcinogenesis 26: 271-279.

Fung ML, Lam SY, Dong X, Chen Y, et al. (2002). Postnatal hypoxemia increases angiotensin II sensitivity and upregulates AT1a angiotensin receptors in rat carotid body chemoreceptors. J. Endocrinol. 173: 305-313.

Fyhrquist F and Saijonmaa O (2008). Renin-angiotensin system revisited. J. Intern. Med. 264: 224-236.

George AJ, Thomas WG and Hannan RD (2010). The renin-angiotensin system and cancer: old dog, new tricks. Nat. Rev. Cancer 10: 745-759.

Gallagher PE and Tallant EA (2004). Inhibition of human lung cancer cell growth by angiotensin-(1-7). Carcinogenesis 25: 2045-2052.

Gallagher PE, Cook K, Soto-Pantoja D, Menon J, et al. (2011). Angiotensin peptides and lung cancer. Curr. Cancer Drug Targets 11: 394-404.

Imai N, Hashimoto T, Kihara M, Yoshida S, et al. (2007). Roles for host and tumor angiotensin II type 1 receptor in tumor growth and tumor-associated angiogenesis. Lab. Invest. 87: 189-198.

Iwai M and Horiuchi M. (2009). Devil and angel in the renin-angiotensin system: ACE-angiotensin II-AT1 receptor axis vs. ACE2-angiotensin-(1-7)-Mas receptor axis. Hypertens Res.32: 533-536.

Jemal A, Bray F, Center MM, Ferlay J, et al. (2011). Global cancer statistics. CA Cancer J. Clin. 61: 69-90.

Kanehira T, Tani T, Takagi T, Nakano Y, et al. (2005). Angiotensin II type 2 receptor gene deficiency attenuates susceptibility to tobacco-specific nitrosamine-induced lung tumorigenesis: involvement of transforming growth factor-beta-dependent cell growth attenuation. Cancer Res. 65: 7660-7665.

Kinoshita J, Fushida S, Harada S, Yagi Y, et al. (2009). Local angiotensin II-generation in human gastric cancer: correlation with tumor progression through the activation of ERK1/2, NF-kappaB and survivin. Int. J. Oncol. 34: 1573-1582.

Koka V, Huang XR, Chung AC, Wang W, et al. (2008). Angiotensin II up-regulates angiotensin I-converting enzyme (ACE), but down-regulates ACE2 via the AT1-ERK/p38 MAP kinase pathway. Am. J. Pathol. 172: 1174-1183.

Mao C, Hou J, Ge J, Hu Y, et al. (2010). Changes of renal AT1/AT2 receptors and structures in ovine fetuses following exposure to long-term hypoxia. Am. J. Nephrol. 31: 141-150.

Neo JH, Ager EI, Angus PW, Zhu J, et al. (2010). Changes in the renin angiotensin system during the development of colorectal cancer liver metastases. BMC Cancer 10: 134.

Ni L, Feng Y, Wan H, Ma Q, et al. (2012). Angiotensin-(1-7) inhibits the migration and invasion of A549 human lung adenocarcinoma cells through inactivation of the PI3K/Akt and MAPK signaling pathways. Oncol. Rep. 27: 783-790.

Pickel L, Matsuzuka T, Doi C, Ayuzawa R, et al. (2010). Overexpression of angiotensin II type 2 receptor gene induces cell death in lung adenocarcinoma cells. Cancer Biol. Ther. 9: 4.

Rocken C, Rohl FW, Diebler E, Lendeckel U, et al. (2007). The angiotensin II/angiotensin II receptor system correlates with nodal spread in intestinal type gastric cancer. Cancer Epidemiol. Biomarkers Prev. 16: 1206-1212.

Shibata K, Makino I, Shibaguchi H, Niwa M, et al. (1997). Up-regulation of angiotensin type 2 receptor mRNA by angiotensin II in rat cortical cells. Biochem. Biophys. Res. Commun. 239: 633-637.

Soto-Pantoja DR, Menon J, Gallagher PE and Tallant EA (2009). Angiotensin-(1-7) inhibits tumor angiogenesis in human lung cancer xenografts with a reduction in vascular endothelial growth factor. Mol. Cancer Ther. 8: 1676-1683. 
Suganuma T, Ino K, Shibata K, Kajiyama H, et al. (2005). Functional expression of the angiotensin II type 1 receptor in human ovarian carcinoma cells and its blockade therapy resulting in suppression of tumor invasion, angiogenesis, and peritoneal dissemination. Clin. Cancer Res. 11: 2686-2694.

Tanaka M, Tsuchida S, Imai T, Fujii N, et al. (1999). Vascular response to angiotensin II is exaggerated through an upregulation of AT1 receptor in AT2 knockout mice. Biochem. Biophys. Res. Commun. 258: 194-198.

van Kats JP, Danser AH, van Meegen JR, Sassen LM, et al. (1998). Angiotensin production by the heart: a quantitative study in pigs with the use of radiolabeled angiotensin infusions. Circulation 98: 73-81.

Volpe M, Musumeci B, De Paolis P, Savoia C, et al. (2003). Angiotensin II AT2 receptor subtype: an uprising frontier in cardiovascular disease? J. Hypertens. 21: 1429-1443.

Zhang R, Wu Y, Zhao M, Liu C, et al. (2009). Role of HIF-1alpha in the regulation ACE and ACE2 expression in hypoxic human pulmonary artery smooth muscle cells. Am. J. Physiol. Lung Cell. Mol. Physiol. 297: 10. 